Historic, Archive Document

Do not assume content reflects current scientific knowledge, policies, or practices. 



\section{WHOLESALE PRICE LIST}

of

Perennial, Rock and Alpine Plants

\section{HURLSTONE NURSERIES, Inc. BRIARCLIFF MANOR,} Tel., Briarcliff 1505 NEW YORK

\section{Notice to Customers}

Not less than six of a variety will be supplied at the dozen rate; nor less than fifty of a variety at the hundred rate.

All goods are carefully packed, in boxes, for which we charge for at cost.

After delivering to the forwarders, all goods are entirely at the risk of the purchaser. For any loss or delay in transit the forwarders alone must be held responsible. All quotations are F. O. B. Briarcliff Manor.

Orders from unknown parties must be accompanied by cheque or money order.

Claims, if any, must be made within 10 days after receipt of goods.

We warrant all stock to be true to name, with the explicit understanding and igreement with the purchaser that should any prove not true to name we will refund the money paid or replace with other stock, but we shall not be held liable for damages beyond this.

\section{HARDY PERENNIAL PLANTS STRONG FIELD GROWN}

ACHILLEA, The Pearl, white ......................... 1.00

ALYSSUM, Saxatile Compactum, yellow ............... 1.00

ANCHUSA, Dropmore, blue ............................ 1.00

ANTHEMIS, Tinctora, golden yellow ..................... 1.00

AQUILEGIA, Mrs. Scott Elliott, long spurred............. 1.00

New Rose, rose-scarlet ..................... 1.25

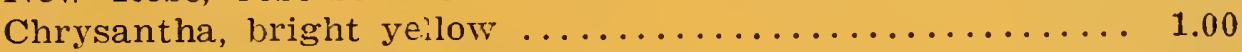

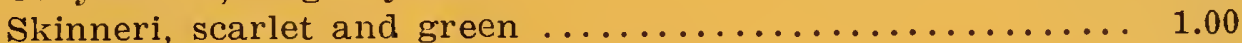

ANEMONE JAPANICA, Whirlwind $\ldots \ldots \ldots \ldots \ldots \ldots \ldots \ldots . \ldots \ldots$

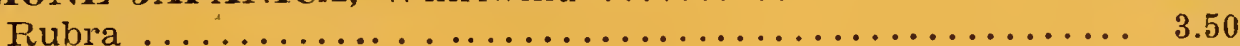

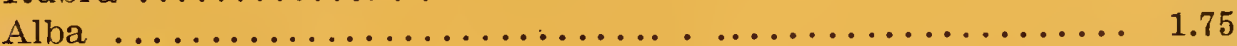

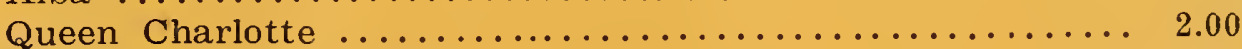

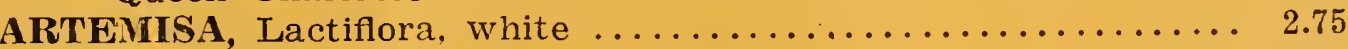

ARMERIA or SEA PINK, bright pink $\ldots \ldots \ldots \ldots \ldots \ldots \ldots \ldots 2.25$

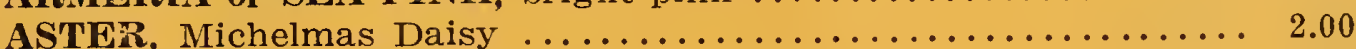

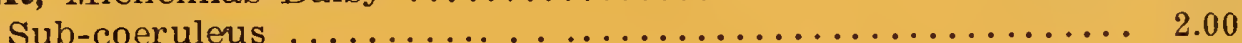

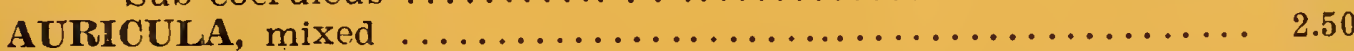

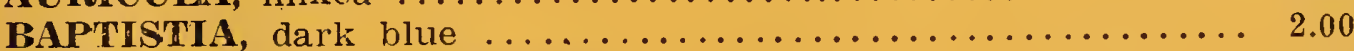

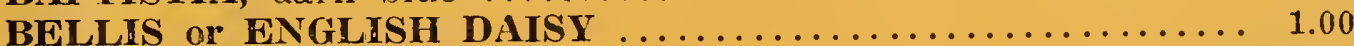

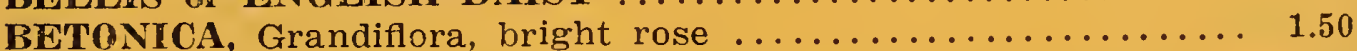

BOCCONIA or PLUME POPPY, creamy white............ 1.50

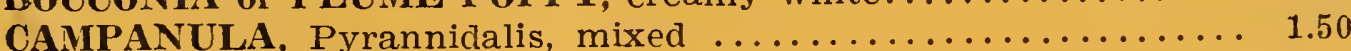

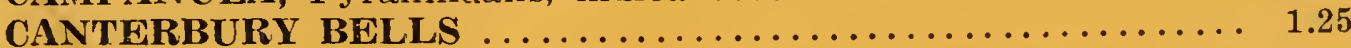




\section{HARDY PERENNIAL PLANTS—Continued}

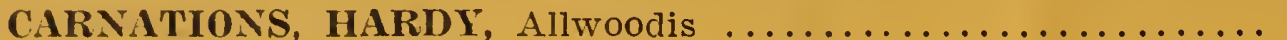

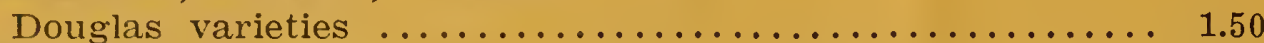

10.00

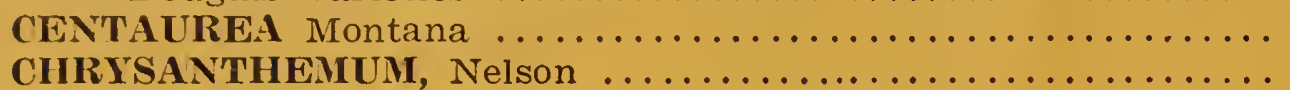

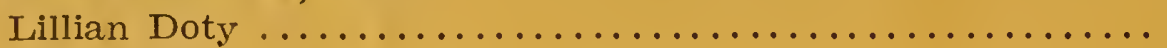

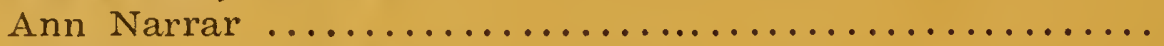

Donald

1.50

10.00

10.00

$1.00 \quad 7.00$

$1.00 \quad 7.00$

$1.00 \quad 7.00$

$1.00 \quad 7.00$

CIIELONE Lyonii, purplish red ..................... 1.75

12.00

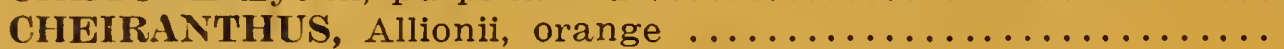

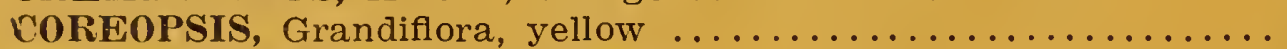

DELPHINIUM, English hybrids $\ldots \ldots \ldots \ldots \ldots \ldots \ldots \ldots \ldots$

DIANTHUS Barbatus, $\operatorname{mixed} \ldots \ldots \ldots \ldots \ldots \ldots \ldots \ldots \ldots \ldots \ldots$ Heddewigii

2.00

1.00

2.00

15.00

6.00

15.00

6.00

10.00

DIGITALIS Gloxiniaefiora, rose to deep purple $\ldots \ldots \ldots \ldots \ldots \ldots$ Alba, white $\ldots \ldots \ldots \ldots \ldots \ldots \ldots \ldots \ldots \ldots \ldots \ldots \ldots \ldots$

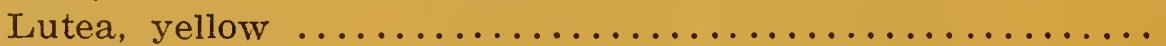

ERINUS, Alpinus, rosy purple $\ldots \ldots \ldots \ldots \ldots \ldots \ldots \ldots \ldots \ldots$

GAILLARDIA, the King $\ldots \ldots \ldots \ldots \ldots \ldots \ldots \ldots \ldots \ldots \ldots$

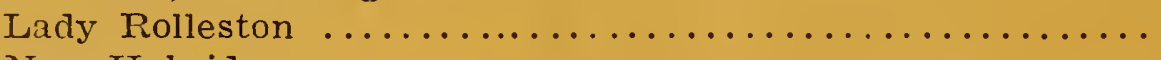

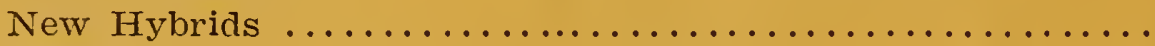

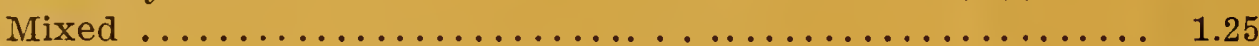

GEUM, Mrs. Bradshaw, fiery red ................... 1.50

Lady Stratheden, golden yellow ................. 2.50

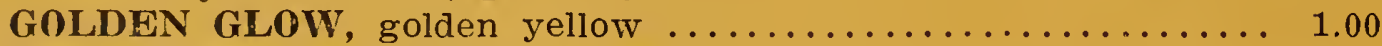

GYPSOPHILA, Paniculata Compacta ................. 1.25

HEMEROCALLIS or DAY LILY, yellow and orange varieties . . . 2.50

HOLLYHOCK, Chater's Double, mixed ................ 1.00

IBERIS Gibraltarica, pale lilac ..................... 1.50

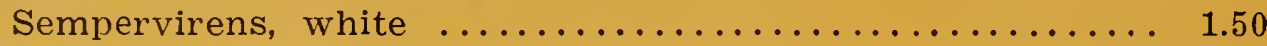

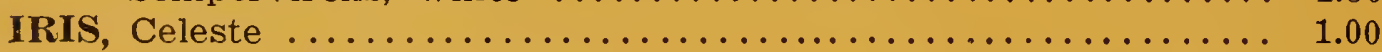

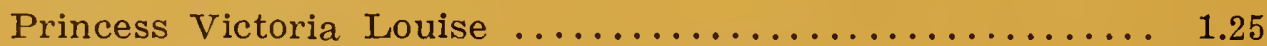

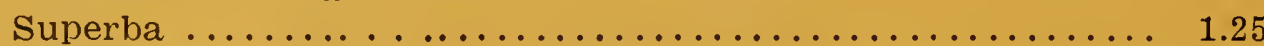

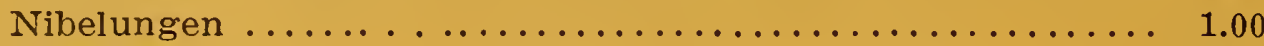

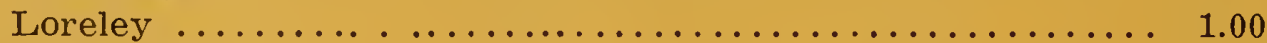

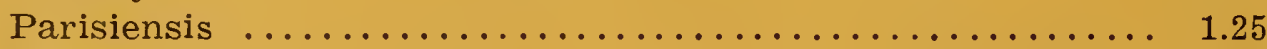

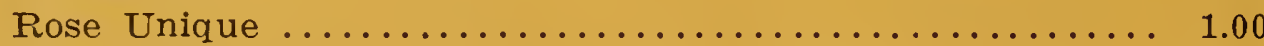

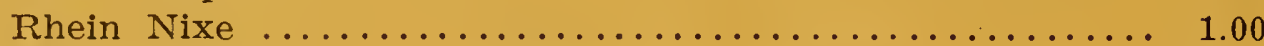

Mme. Chereau ............................. 1.00

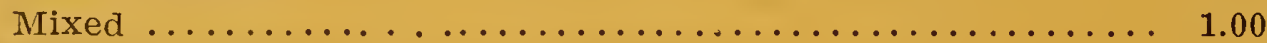

6.00

6.00

6.00

25.00

8.00

8.00

8.00

8.00

10.00

18.00

6.00

7.00

6.00

10.00

10.00

5.00

8.00

8.00

5.00

5.00

8.00

5.00

5.00

5.00

5.00

12.00

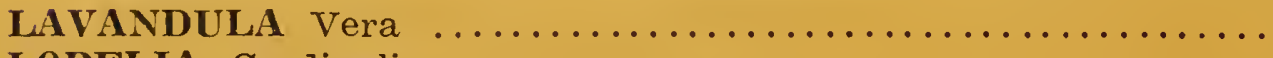

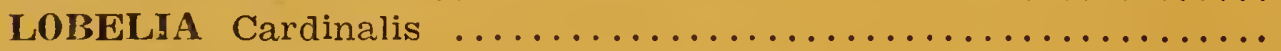

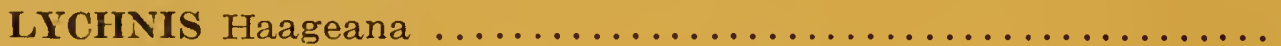

1.75

1.50

1.50

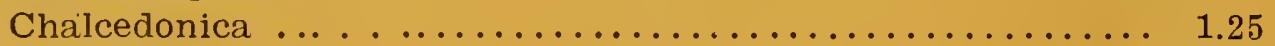

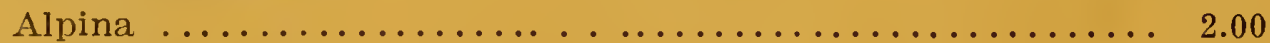

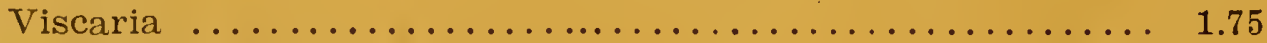

10.00

10.00

8.00

15.00

12.00

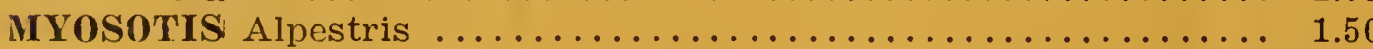

$1.50 \quad 10.00$

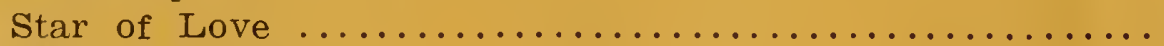

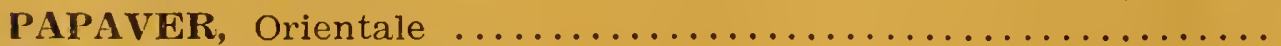

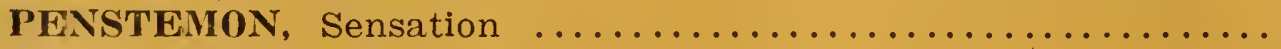

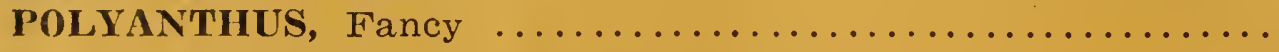

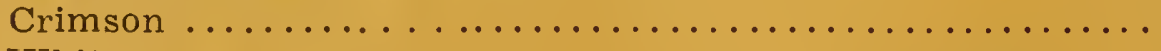

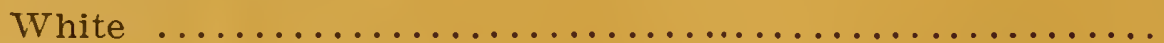

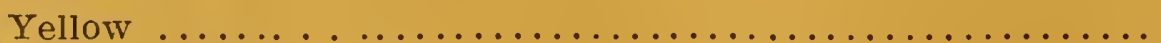

$1.50 \quad 10.00$

$1.50 \quad 10.00$

$1.50 \quad 12.00$

$1.50 \quad 12.00$

$1.50 \quad 12.00$

$1.50 \quad 12.90$

$1.50 \quad 12.00$

$2.00 \quad 15.00$

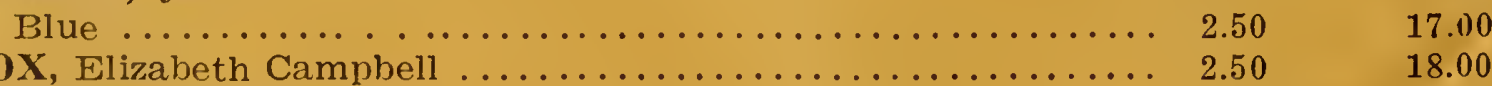

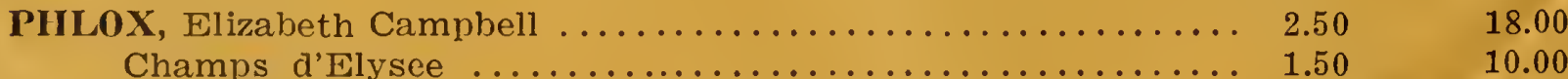




\section{HARDY PERENNIAL PLANTS-Continued}

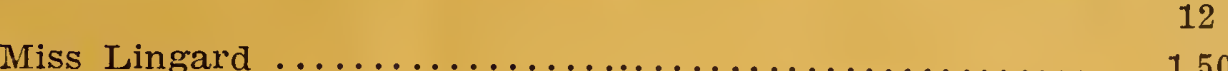

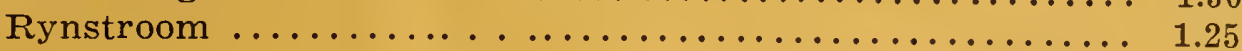

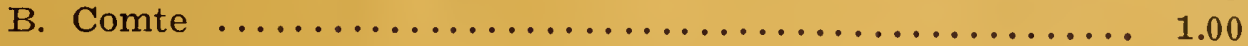

10.00

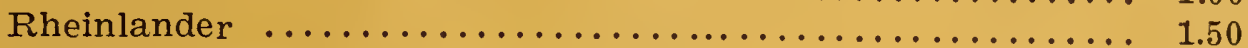

Subulata, moss pink, white and lilac ................ 1.50

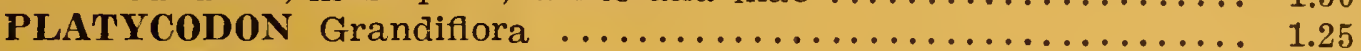

10.00

10.00

8.00

8.00

12.00

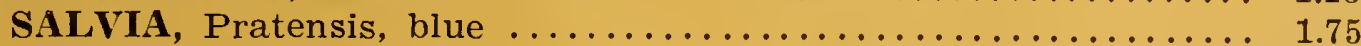

SCABIOSA Caucasica ............................... 2.00

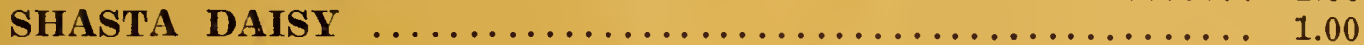

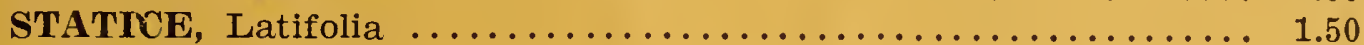

STOKESIA, Cyanea, mixed ............................ 1.50

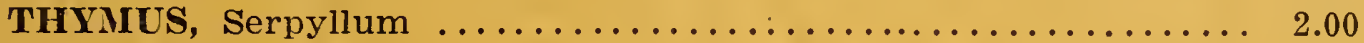

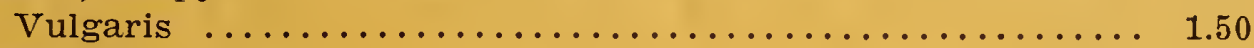

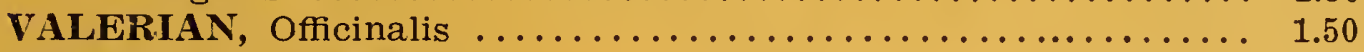

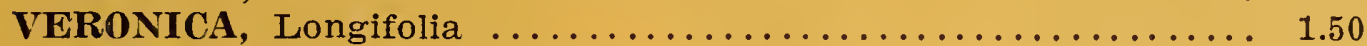

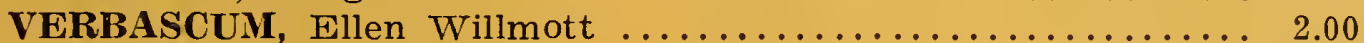

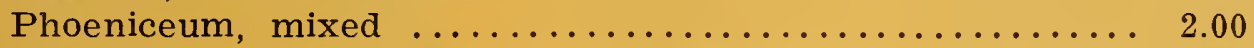

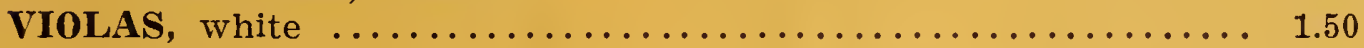

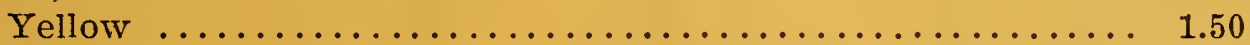

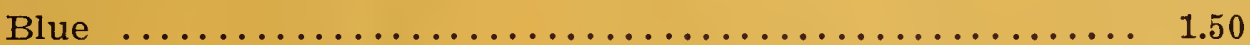

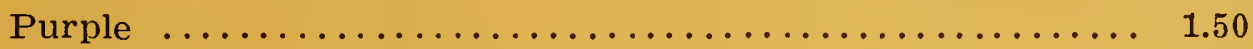

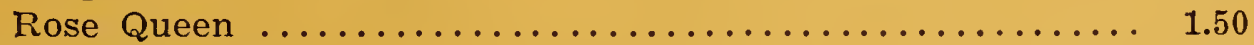

Cornuta, white .............................. 1.50

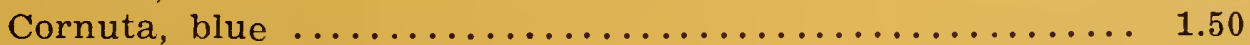

Cornuta, blue, Gold Medal ....................... 1.50

Cornuta, Papilio ............................ 1.50

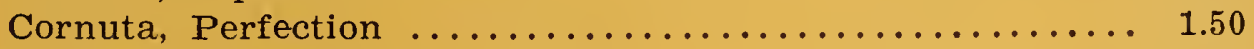

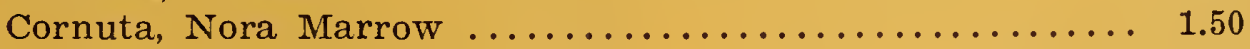

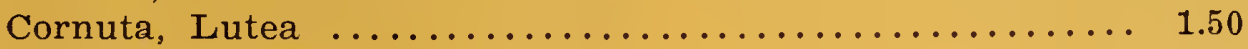

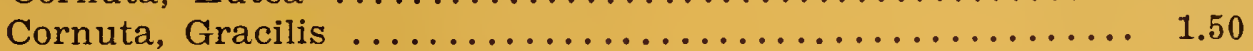

\section{ROCK and ALPINE PLANTS}

\section{$\$ 1.50$ per dozen, $\$ 10.00$ per 100 except as otherwise noted}

ALYSSUM Saxatile Compactum

AETHIONEMA Grandiflora, $\$ 3.50$ per dozen, $\$ 18.00$ per 100 .

ANTHEMIS Montana

ASTER, Alpinum Alba

AUBRETIA, Bougainaili

Deltoides

Grecea

Hendersonii

Leechlini

New Hybrids

Mixed

Lloyd Edward

Bridesmaid

Purpurea

ARABIS, Alpina Superba

ARUILEGIA, Glandulosa

ACHILLES, Tomentosa.

AURICULA, Alpina, $\$ 2.50$ per dozen, $\$ 12.00$ per 100

ACHILLEA, Rupestris 
CAMPANULA, Carpatica, alba, white Carpatica, blue Persifolia, alba

CYTISIS, Albus, $\$ 2.50$ per dozen, $\$ 12$ per 100

CHEIRANTIUS, Linifolius

CERASTIUM, Grandeflorum

DIANTHUS, Heddewegii

Rock Pink

Arenarius

Caesius

Neglectus

DRABA AIZOIDES

DELPHINIUM, Nudicaule

ERIGERON, Aurantiacus

ERINUS, Alpinus

ERYSIMUM, Rupestre, $\$ 2.00$ per dozen, $\$ 15.00$ per 100

GENISTA, Andreana

GENTIANA

GERANIUM, Sanguineum

HELIANTHEMUMS, $\$ 2.50$ per dozen, $\$ 12.00$ per 100

HEUCHERA, Sanguinea

HIERADIUMI, Aurantiacum

IBERIS

IRIS, Forresti, $\$ 2.50$ per dozen, $\$ 12.00$ per 100

Pumile, $\$ 2.50$ per dozen, $\$ 12.00$ per 100

Siberica, $\$ 2.50$ per dozen, $\$ 12.00$ per 100

Snow Queen, $\$ 2.50$ per dozen, $\$ 12.00$ per 100

LAVANDULA VERA

LEONTOPODIUN, Alpinum

LYCHNIS, Alpina

Haageana

Viscaria, $\$ 2.50$ per dozen, $\$ 12.00$ per 100

Largascae, $\$ 2.50$ per dozen, $\$ 12.00$ per 100

LINUM, Augustifolium

PHLOX, Subulato, rose White

Lilac

PAPAVER, Alpinum, $\$ 2.50$ per dozen, $\$ 12.00$ per 100 Burseri, $\$ 2.50$ per dozen, $\$ 12$ per 100

Nudicaule Album, $\$ 2.50$ per dozen, $\$ 12.00$ per 100

POTENTILLA, Rupestre

Pyrenaica

Alchemilloides

SILINE Pendula Compacta (annual)

SEDUM Acre

Coeruleum

SAPONARIA Oxyomoides

SIVEET ROCKET

SAXIFRAGA, Mixed

Longfolia, $\$ 3.00$ per dozen, $\$ 15.00$ per 100

Rhei Superba, $\$ 3.00$ per dozen, $\$ 15.00$ per 100

SILENE Alpestris

SILENE Rupestris

SEDUM Aizoon

SCABIOSA Vestina, $\$ 3.00$ per dozen, $\$ 15.00$ per 100

VIOLAS (See Perennial List) 